\title{
Action of an Indolinone Derivative on Plasma Hemostasis
}

\author{
Bykov VV', Serebrov VYu' ${ }^{1}$, Udut $\mathrm{EV}^{1}$ and $U d u t \mathrm{VV}^{2,3 *}$ \\ 1Siberian State Medical University (SSMU) of Russian Ministry of Health, Biochemistry and Molecular Biology Faculty, Russia \\ ${ }^{2}$ Tomsk National Research Medical Center of the Russian Academy of Sciences, Goldberg Research Institute of Pharmacology and Regenerative \\ Medicine, Russia \\ ${ }^{3}$ National Research Tomsk State University
}

${ }^{\star}$ Corresponding author: V.V. Udut, Professor, Tomsk National Research Medical Center of the Russian Academy of Sciences, Goldberg Research Institute of Pharmacology and Regenerative Medicine, Russia; Email: udutv@mail.ru

Received: March 27, 2019; Accepted: April 09, 2019; Published: April 12, 2019;

\begin{abstract}
The action of a new pharmaceutical substance of indolinone series, an sGC inducer with antiplatelet activity, on rat blood plasma hemostasis was studied. It was shown that the antiplatelet substance after single oral administration to rats considerably increases thrombin time after 3 hours $(24.5$ versus 17.3 in control, $\mathrm{p}<0.05)$. Other plasma hemostasis parameters were unchanged.
\end{abstract}

Key words: antiplatelet, indolinone derivative, plasma hemostasis.

\section{Introduction}

Thrombosis plays a key role in the development of acute coronary syndrome, making antiplatelet therapy an important part of prevention and treatment of cardiovascular diseases [1]. One of the main risk factory of cardiovascular disease relapse is insufficiency of existing therapy in people resistant to aspirin and clopidogrel $[2,3]$. This problem can be solved by using antiplatelet drugs with a novel mechanism of action. Also, increased blood clotting plays a role in cardiovascular complications $[4,5]$. There were no earlier studies of the action of the indolinone derivative on blood plasma hemostasis.

Study goal - Assess the action of a new antiplatelet compound on rat blood plasma hemostasis after oral administration.

\section{Materials and Methods}

Test article - pharmaceutical substance 2-[2-[(5RS)-5(hydroxymethyl)-3-methyl-1,3-oxazolidine-2-yliden ]-2cyanoethylidene]-1H-indol-3(2H)-one.

Outbred Wistar rats $(\mathrm{n}=10)$ were used as test system. Rat handling was performed in accordance with the European Convention and other regulating documents [6].

Intact blood was sampled from common carotid artery of anesthetized rats. The blood was stabilized with $3.8 \%$ solution of sodium citrate in 9:1 (v:v) ratio, blood plasma was produced by centrifuging at $2000 \mathrm{~g}$ for $20 \mathrm{~min}$.

Plasma hemostasis was assessed by fibrinogen content, activated Partial Thromboplastin Time (aPTT), Prothrombin Time (PT) and Thrombin Time (TT). These parameters were assessed by KG-4 coagulometer (Cormay, Poland). Fibrinogen content was assessed using Claus method.
The antiplatelet drugs were administered to rats once orally in $10 \mathrm{mg} /$ $\mathrm{kg}$ dose. At the end of experiment the animals were sacrificed by $\mathrm{CO}_{2}$.

Statistical analysis was performed by «R» software. The data is presented as mean values and mean standard deviation $(\mathrm{M} \pm \mathrm{m})$. Significance of difference $(\mathrm{p}<0.05)$ between the tests was assessed using Mann-Whitney U test.

\section{Result and Discussion}

No significant changes of main parameters of plasma hemostasis: fibrinogen, aPTT and PT, were found 3 hours after single oral administration of the antiplatelet drug in $10 \mathrm{mg} / \mathrm{kg}$ dose. TT was increased by $42 \%$ compared to the control group (Table 1 ).

Table 1. Effects of the new antiplatelet drug $(10 \mathrm{mg} / \mathrm{kg})$ on rat blood plasma hemostasis.

\begin{tabular}{|l|c|c|c|c|}
\hline Group & Fibrinogen & aPTT & PT & TT \\
\hline Control (n=5) & $2.0 \pm 0.1$ & $14.8 \pm 0.7$ & $20.4 \pm 0.6$ & $17.3 \pm 2.3$ \\
\hline Antiplatelet drug (n=5) & $1.8 \pm 0.1$ & $15.2 \pm 0.6$ & $20.1 \pm 0.3$ & $24.5 \pm 1.0^{*}$ \\
\hline
\end{tabular}

Note: ${ }^{*}-\mathrm{p}<0.05$ compared to control.

The new antiplatelet drug doesn't affect blood plasma fibrinogen content, activated partial thromboplastin time and prothrombin time, but considerably increases the thrombin time, 3 hours after single oral administration in $10 \mathrm{mg} / \mathrm{kg}$ dose [7]. The reduction of platelet aggregation by the new drug leads to reduced exit of active components of the coagulation system from the platelets, which may account for the lengthening of thrombin time. There may also be other explanations for this. Additional studies are required for confirmation or discovery of another mechanism, including, possibly, direct inhibition of thrombin. 


\section{References}

1. Popova LV, Axenova MB, Khlevchuk TB (2016) Antiplatelet activity in cardiology. Clinical medicine 10: 729-36.

2. Shantsila E, Lip GY (2009) Variability of response to antiplatelet therapy: what should we do next? Fundam Clin Pharmacol 23: 19-22. [crossref]

3. Lee PY, Chen WH, Ng W, Cheng X, Kwok JY, et al. (2005) Low-dose aspirin increases aspirin resistance in patients with coronary artery disease. Am J Med 118: 723-727. [crossref]

4. Frere C, Cuisset T, Quilici J, Camoin L, Carvajal J, et al. (2007) ADP-induced platelet aggregation and platelet reactivity index VASP are good predictive markers for clinical outcomes in non-ST elevation acute coronary syndrome. Thromb Haemost 98: 838-843. [crossref]

5. Patrono C (2003) Aspirin resistance: definition, mechanisms and clinical readouts. J Thromb Haemost 1: 1710-1713. [crossref]

6. Carkishenko NN, Grachev SV (2003) Guidelines on laboratory animals and alternative models in biomedical technology. Profile: Moscow.

7. Triplett DA (2000) Coagulation and bleeding disorders: review and update. Clin Chem 46: 1260-1269. [crossref] 